\title{
A VEGETAÇÃO DE CAPOEIRA NA REGIÃO DO CURSO MÉDIO DO RIO JACUI, RS
}

Josē Newton Cardoso Marchiori, Solon J. Longhi e Miguel A. Durlo Departamento de Ciēncias Florestais. Centro de Ciēncias Rurais. UFSM. Santa Maria, RS.

RESUMO

o trabalho descreve a composição florística e estrutura da vegetação de capoeira na região do curso médio do Rio Jacuí, no es tado do Rio Grande do Sul. Este tipo florestal é altamente heterogê neo. Foram encontradas 45 espécies lenhosas, das quais são mais abun dantes as plantas pioneiras. Espēcies de importância econômica ocor rem com menor abundância. Foram tambēm identificados 96 espēcies não lenhosas.

SUMMARY

MARCHIORI, J.N.C.; LONGHI,S.J. and DURLO,M.A., 1982. Brushwood vegetation in the region of the mid flow of the Jacuí River, RS.Ciên cia e Natura (4):141-150.

The work describes the floristic composition and structure of the brushwood vegetation in the region of the mid flow of the Ja cuí River, RS. This forest type is highly heterogeneous. Forty five wood species were found, of which the pioneer vegetation is the most abundant. Species of economic importance occur less frequently. Also identified were ninety-six non-wood species.

INTRODUÇAO

As informações científicas sobre a estrutura e composição da vegetação florestal nativa são escassas no Rio Grande do Sul. A maior parte das matas nativas foram devastadas com a colonização e utilização das terras para a agricultura e pecuāria. Restam, atual mente, poucas āreas com vegetação primitiva intacta.

0 abandono de āreas de cultivo em regiões originalmente co bertas por matas proporciona um lento processo de reconstituição des ta vegetação. Atualmente, a maior parte da vegetação florestal nat $\underline{i}$ va encontra-se em diferentes estāgios de sucessão.

0 vale do curso médio do Rio Jacuí, originalmente coberto por densa mata subtropical, sofreu as transformações acima menciona das. Para esta região foram reconhecidos trēs tipos de vegetação flo restal: capoeira, mata secundāria e mata ribeirinha.

0 presente estudo tem o objetivo de caracterizar a estru tura e composição da formação florestal "capoeira" encontrada nesta região. 
Por estrutura de uma vegetação compreende-se 0 agregado quantitativo de unidades funcionais, ou seja, a ocupação espacialdos componentes de uma massa vegetal. Para a sua determinação ē necessā rio reconhecer a quantidade ou percentagem dos indivíduos de cada es pēcie representada na vegetação (DANSEREAU, citado por MONTOYA MA QUIN, 8).

GOLDSTEIN \& GRINGAL (4) afirmam que o estudo da estrutura consiste na organização dos vegetais em agrupamentos atravēs da anā lise botānica e distribuição espacial das espēcies.

Segundo FÖRSTER (3), a anālise estrutural baseada em parā metros mensurāveis tem a vantagem de possibilitar a comparação entre tipos diferentes de vegetação.

KELLMAN (6) reconhece tambēm a importāncia de parāmetros numēricos, tais como os valores de abundāncia, frequência e dominān cia para a caracterização de tipo florestal, pois considera que as descrições puramente fisionōmicas não descrevem satisfatoriamente a estrutura da vegetação.

A abundância das espécies constituintes de uma vegetação, associada a caracteres fisionómicos e florísticos, são também reco mendados, entre outros, por CAINE \& CASTRO (1), LAMPRECHT (7) e FINOL (2).

\section{CARACTERISTICAS DA REGIAOO}

o presente estudo foi executado na ārea de inundação da fü tura barragem de Dona Francisca, no Rio Jacuí, Rio Grande do Sul.

Nesta região, os rios Jacuí e Jacuizinho formam vales es treitos e topografia acidentada. Em sua maior parte, os solos apre sentam afloramentos de rochas, havendo a ocorrência de deposições aluviais nos raios internos das curvas descritas pelo rio Jacuí. de Köppen.

o clima è do tipo cfa, segundo a classificação climātica

A vegetação original da região faz parte da mata úmida sub tropical perenifólia do Sul do Brasil. De acordo com HUECK (5), tra ta-se de uma floresta muito heterogēnea e rica em espēcies de impor tância florestal, apresentando grande densidade de vegetação, lia nas e epífitas, bem como samambaias arborescentes em alguns lugares.

\section{LEVANTAMENTO DA VEGETAÇAOO}

Como capoeira, definiu-se diferentes tipos fisionômicos de vegetação florestal, resultantes de estāgios sucessionais distintos. A capoeira foi caracterizada como uma vegetação de estrutura menos desenvolvida do que a Mata Secundäria, e de composição, estrutura e dinâmica dependentes da utilização anterior da ārea e do seu tempo 
de abandono.

Para caracterizar a vegetação constituinte da capoeira, fo ram utilizadas 10 amostras de $9 \mathrm{~m}^{2}(3 \times 3 \mathrm{~m})$, distribuíidas aleatoria mente na ārea de estudo.

Em cada amostra foram identificadas e contadas as espécies arbōreas e herbáceas, bem como as regenerações de espécies lenhosas.

RESULTADOS E DISCUSSAOO

As espécies lenhosas encontradas neste tipo florestal es tão enumeradas na Tabela I. Estas espécies distribuem-se em 42 gêne ros de 22 famílias botânicas.

TABELA I. NOME CIENTIFICO E FAMILIA BOTANICA DAS ESPECIES LENHOSAS.

\begin{tabular}{|c|c|c|}
\hline Nọ & NOME CIENTIFICO & FAMILIA \\
\hline 1 & Actinostemon concolor (Spreng.) Mull. Arg. & Euphorbiaceae \\
\hline 2 & Allophylus edulis (Camb.) Radlk. & Sopindaceae \\
\hline 3 & Aloysia sellowii (Briq.) Mold. & Verbenaceae \\
\hline 4 & Balfourodendron riedelianum (Engler) Engler & Rutaceae \\
\hline 5 & Bauhinia candicans Benth. & Leguminosae \\
\hline 6 & Blepharocalyx angustifolius Berg & Myrtaceae \\
\hline 7 & Brittoa guazumaefolia (Camb.) Legr. & Myrtaceae \\
\hline 8 & Compomanesia xanthocarpa (Mart.) Berg & Myrtaceae \\
\hline 9 & Casearia silvestris SW. & Flacourtiaceae \\
\hline 10 & Cestrum calycinum willd. & Solanaceae \\
\hline 11 & ChrysophyZZum gonocarpum (Mart. \& Eich1.) Engl. & Sopotaceae \\
\hline 12 & Chrysophy Zzum marginatum (Hook. \& Arn.) Radlk. & Sopotaceae \\
\hline 13 & Cordia ecalyculata Vell. & Boraginaceae \\
\hline 14 & Cupania vermalis Camb. & Sapindaceae \\
\hline 15 & Dalbergia frutescens (Vel1.) Britt. & Legrominosae \\
\hline 16 & Erythrina falcata Benth. & Legrominosae \\
\hline 17 & Eugenia uniflora $\mathrm{L}$. & Myrtaceae \\
\hline 18 & Fagara rhoifolia (Lam.) Engler & Rutaceae \\
\hline 19 & Feijoa selzowiana Berg & Myrtaceae \\
\hline 20 & Helietta apiculata Benth. & Rutaceae \\
\hline 21 & Inga marginata willd. & Legrominosae \\
\hline 22 & Luehea divaricata Mart. & Tiliaceae \\
\hline 23 & Maba incons tans (Jacq.) Griseb. & Ebenaceae \\
\hline 24 & Machaerion stipitation (DC.) Vog. & Leguminosae \\
\hline 25 & Manihot grahami Hook. & Euphorbiaceae \\
\hline 26 & Matayba elaeagnoides Radlk. & Sopindaceae \\
\hline 27 & Maytenus ilicifolia Mart. & Celastraceae \\
\hline 28 & Myrocarpus frondosus Fr. Allen. & Leguminosae \\
\hline
\end{tabular}


TABELA I. Continuação.

\begin{tabular}{lll}
\hline \hline No & \multicolumn{1}{c}{ NOME CIENTIFICO } & FAMILIA \\
\hline 29 & Nectandra megapotamica (Spreng.) Nees & Lauraceae \\
30 & Ocotea puberula Nees & Lauraceae \\
31 & Parapiptadenia migida (Benth.) Brenan & Leguminosae \\
32 & Patagonula americana L. & Boraginaceae \\
33 & Psychotria cartaginensis Jacq. & Rubiaceae \\
34 & Sebastiania brasiliensis Spreng. & Eiphorbiaceae \\
35 & Seguieria guaranitica Speg. & Phytolacaceae \\
36 & Solanum paniculatim L. & Solanaceae \\
37 & Solanum verbascifolium L. & Solanaceae \\
38 & Sorocea bomplandii (Baill.) Burger & Moraceae \\
39 & Strychnos brasiliensis (Spreng.) Mart. & Logoniaceae \\
40 & Tabebuia cvellanedae Lorentz ex Giseb. & Bignoniaceae \\
41 & Tecoma stans (L.) Kunth & Bignoniaceae \\
42 & Trema micrantha (L.) Blume & Ulmaceae \\
43 & Trichilia catigua A. Juss. & Meliaceae \\
44 & Trichilia elegans A. Juss. & Meliaceae \\
45 & Villaresia paniculata (Mart.) Miers & Icacinaceae \\
\hline
\end{tabular}

Um dos tipos fisionōmicos que se destacam em estāgios su cessionais iniciais de capoeira ē resultante da associação de Trema micrantha e Tecoma stans, formação encontrada notadamente em locais mais inclinados e pedregosos. Em estāgios mais evoluídos, observa-se uma heterogeneidade crescente, concomitante com a redução da abundân cia destas duas espēcies.

Em estāgio intermediārio salientam-se, ao lado das espē cies acima citadas, outras heliōfilas e seletivas xerōfilas, tais co mo Tabebuia avelzanedae, Patagonula americana, Cordia trichotoma, Maytenus ilicifolia, Seguieria guaranitica, e as espécies de sola naceae citadas na Tabela I.

Os estágios sucessionais mais evoluídos de Capoeira, obser vados na região, caracterizam-se pela presença abundante de espécies lenhosas umbrófilas, indicando seu carāter de transição para a Mata Secundāria.

As espēcies não lenhosas componentes da vegetação de Ca poeira, em nūmero de 96 , são apresentadas na Tabela II.

A abundância em espécies não lenhosas é característica dos estāgios iniciais da sucessão vegetal, e se explica pela grande va riedade de condições ecolōgicas locais, o que possibilita a ocorrên cia de espécies de exigências bastante diferentes. 
TABELA II. NOME CIENTIFICO E FAMILIA BOTÃNiCA DAS ESPECIES NAOO LENHOSAS.

\begin{tabular}{|c|c|c|}
\hline Nọ & NOME CIENTIFICO & FAMILIA \\
\hline 1 & Abutilon pictum (Gill). Walp. & Malvaceae \\
\hline 2 & Acalypha gracilis Spreng. & Euphorbiaceae \\
\hline 3 & Adiantopsis spp. & Polypodiaceae \\
\hline 4. & Adiantrom spp. & Polypodiaceae \\
\hline 5 & Andropogon lateralis Nees & Gramineae \\
\hline 6 & Aneimia phy llitidis (L.) Sw. & Schizaeaceae \\
\hline 7 & Arcajia hortomum Fourn. & Asclepiadaceae \\
\hline 8 & Aristolochia triangularis Cham. \& Schlecht. & Aristolochiaceae \\
\hline 9 & Arrabidaea chica (H. et B.) Verlot & Bignoniaceae \\
\hline 10 & Asclepias curassavica L. & Asclepiadaceae \\
\hline 11 & Bacchamis dracunculifolia DC. & Compositae \\
\hline 12 & Baccharis spp. & Compasitae \\
\hline 13 & Begonia cuculata (L.) Miers & Begoniaceae \\
\hline 14 & Blainvizzea biamistata DC. & Compositae \\
\hline 15 & Blechnum spp. & Polypodiaceae \\
\hline 16 & Bohemeria caudata Sw. & Urtianceae \\
\hline 17 & Bulbostylis capilzaris (L.) C.B. Clarke & Cyperaceae \\
\hline 18 & Bulbostylis sphaerocephala (Boeckl.)C.B. Clarke & Cyperaceae \\
\hline 19 & Canna spp. & Cannaceae \\
\hline 20 & Candiospermum grandiflom Sw. & Sapindaceae \\
\hline 21 & Chaptalia nutans (L.) Hems1. & Compositae \\
\hline 22 & Chloris retusa Lag. & Gromineae \\
\hline 23 & Chusquea romosissima Lindm. & Gromineae \\
\hline 24 & Ciclopogon spp. & Orchidaceae \\
\hline 25 & Cisscompe los pareira L. & Menispermaceae \\
\hline 26 & Cyperus spp. & Cyperaceae \\
\hline 27 & Dalechampia micromerià Baill. & Euphorbiaceae \\
\hline 28 & Desmodirom affine Schlecht. & Leguminosae \\
\hline 29 & Digitaria sanguinalis (L.) Scop. & Gramineae \\
\hline 30 & Digitaria swalleniana Henrard & Gramineae \\
\hline 31 & Dryopteris spp. & Polypodiaceae \\
\hline 32 & Dosantha ungis-cati (L.) Miers & Bignoniaceae \\
\hline 33 & Elephantopus mollis HBK. & Compositae \\
\hline 34 & Erigeron bonariensis $\mathrm{L}$. & Compositae \\
\hline 35 & Eupatorium grande Sch. Bip. & Compositae \\
\hline 36 & Eupatorium inalaefolium HBK. & Compositae \\
\hline 37 & Eupatorium intermedium Spreng. & Compositae \\
\hline 38 & Eupatorium Zaevigatum Camb. & Compositae \\
\hline 39 & Forsteronia thyrsoidea (Vell.) Mull. Arg. & Apocynaceae \\
\hline
\end{tabular}


TABELA II. Continuação.

\begin{tabular}{|c|c|c|}
\hline Nọ & CIENTIFICO & FAMILIA \\
\hline 40 & Guadua trinii (Nees) Rupr. & Gramineae \\
\hline 41 & Hybanthus bigibosus (St. Hil.) Hassl. & Violaceae \\
\hline 42 & Hydrocotyle leueocephala Cham. \& Schlecht. & Umbelliferae \\
\hline 43 & Hyptis Laplacea Benth. & Labiatae \\
\hline 44 & Hyp tis mutabilis (L.C. Rich.) Briq. & Labiatae \\
\hline 45 & Indigofera subffmuticosa Mill. & Legrominosae \\
\hline 46 & Ipomoea grandifolia (Dammer) O'Donell & Campanulaceae \\
\hline 47 & Justicia brasiliana Roth & Acanthaceae \\
\hline 48 & Malaxis histionantha Link & Orchidaceae \\
\hline 49 & Melothria cucumis Vell. & Cucurbitaceae \\
\hline 50 & Metaste Ima aphylza & Asclepiadaceae \\
\hline 51 & Nephrolepsis spp. & Polypodiaceae \\
\hline 52 & Olyra humilis Nees & Gramineae \\
\hline 53 & oplismenus hirtellus (L.) Beauv. & Gramineae \\
\hline 54 & Oplismenus setarius (Lam.) Roem. \& Sch. & Gromineae \\
\hline 55 & Oxalis articulata Savigny & Oxalidaceae \\
\hline 56 & Oxalis malobolba Cav. & Oxalidaceae \\
\hline 57 & Oxalis martiana Zucc. & Oxalidaceae \\
\hline 58 & Pavonia kunthii Guerke & Malvaceae \\
\hline 59 & Pavonia sepium St. Hil. & Malvaceae \\
\hline 60 & Panicum glritinosum Sw. & Gramineae \\
\hline 61 & Paspalum conjugatım Berg & Gramineae \\
\hline 62 & Paspatum dilatatum Poir. & Gromineae \\
\hline 63 & Paspatum umillei Steud. & Gramineae \\
\hline 64 & Passiflora suberosa L. & Passifloraceae \\
\hline 65 & Peperomia blanda (Jacq.) HBK. & Piperaceae \\
\hline 66 & Pilea pubescens Lieberm. & Urticaceae \\
\hline 67 & Piper amalago L. & Piperaceae \\
\hline 68 & Piper gaudchoudianrom Kunth & Piperaceae \\
\hline 69 & Polygonum hydropiperoides Michx. & Polygonaceae \\
\hline 70 & Polypodizm Zanceolatum L. & Polypodiaceae \\
\hline 71 & Polypodium phyllitidis L. & Polypodiaceae \\
\hline 72 & Polypodium repens Aubl. & Polypodiaceae \\
\hline 73 & Polypodirm squamulosum Kaulf. & Polypodiaceae \\
\hline 74 & Porophyllum ruderale (Jacq.) Cass. & Compositae \\
\hline 75 & Pteridirom aquilinum (L.) Kuhn & Polypodiaceae \\
\hline 76 & Pyrostegia venusta Miers & Bignoniaceae \\
\hline 77 & Relbunium hypocarpium (L.) Hems ley & Rubiaceae \\
\hline 78 & Ruellia angustifolia (Ness) Lind. & Acanthaceae \\
\hline
\end{tabular}


TABELA II. Continuação.

\begin{tabular}{lll}
\hline \hline No & \multicolumn{1}{c}{ NOME CIENTIFICO } & FAMILIA \\
\hline 79 & Sarchorachis obtusa (Miq.) Tre1. & Piperaceae \\
80 & Scleria spp. & Cyperaceae \\
81 & Scoparia dulcis L. & Scrophulariaceae \\
82 & Serjania laurotteana Camb. & Sapindaceae \\
83 & Serjania spp. & Sapindaceae \\
84 & Setaria leiantha Hacke1 & Gramineae \\
85 & Sicyos polyacanthos Cogn. & Cucurbitaceae \\
86 & Sida rhombifolia L. & Malvaceae \\
87 & Smitax brasiliensis Spreng. & Liliaceae \\
88 & Smilax spp. & Liliaceae \\
89 & Spathicarpa hastifolia Hook. & Araceae \\
90 & Stachytarpheta cayanensis (Rich.) Vahl & Verbenaceae \\
91 & Talinum paniculatim (Jacq.) Gaertn. & Portulaceaceae \\
92 & Tradescantia fluminensis Ve11. & Commelinaceae \\
93 & Tradescantia virginiona L. & Commelinaceae \\
94 & Tragia volubilis L. & Evphorbiaceae \\
95 & Tripogandra elongata (G.F.Meyer) Wood & Commelinaceae \\
96 & Triumfetta rhomboidea Jacq. & Tiliaceae \\
\hline
\end{tabular}

As familias melhor representadas no estrato inferior são em ordem decrescente: Gramineae, Compositae e Polypodiaceae. A abun dância de gramíneas e compostas é característica da vegetação cam pestre e evidencia a estruturação incipiente da vegetação de capoei ra, especialmente em seus estágios iniciais de sucessão.

A Famîlia Polypodiaceae, por apresentar espécies de exigên cias diversas com relação à luminosidade e umidade, encontra-se for temente representada na vegetação do estrato inferior.

Em capoeiras abertas são ainda abundantes espécies heliōfí las, especialmente as das Famílias Malvaceae, Urticaceae; Labiatae, Asclepiadaceae, Cucurbitaceae, Liliaceae e Tiliaceae. Em associa ções mais evoluĩdas predominam espēcies de Oxalidaceae, Piperaceae, Acanthaceae, Violaceae e Rubiaceae.

As espēcies lenhosas mais abundantes na vegetação de Capoei ra são Cestrum calycinum, Allophylus edulis, Parapiptadenia rigida, Trichilia catigua e Inga marginata (Tabela III).

Tratam-se, com excessão de Parapiptadenia rigida, de especies de importância econômica secundāria.

As espécies de maior importância econômica são pouco abun dantes na vegetação de Capoeira. A dinâmica da Mata Subtropical con 
duz, entretanto, a um gradativo aumento da presença de ārvores de grande porte, componentes dos estratos superiores, à medída que a sucessão vegetal progride em direção ao climax.

TABELA III. ABUNDĀNCIA RELATIVA DAS ESPECIES LENHOSAS (\%).

\begin{tabular}{|c|c|c|c|}
\hline Nọ & ESPECIES & ABUNDĀNCIA & RELATIVA \\
\hline 1 & Actinostemom concolor & 0,62 & \\
\hline 2 & Alzophylus edulis & 9,07 & \\
\hline 3 & Balfourodendron riedelianum & 1,04 & \\
\hline 4 & Bauhinia candicans & 3,71 & \\
\hline 5 & Brittoa guazumaefolia & 3,30 & \\
\hline 6 & Campomanesia xanthocarpa & 1,44 & \\
\hline 7 & Casearia sizvestris & 3,09 & \\
\hline 8 & Cestrum calycinum & 11,34 & \\
\hline 9 & Chrysophyzzum gonocarpum & 1,03 & \\
\hline 10 & Chrysophyzzum marginatum & 0,21 & \\
\hline 11 & Cordia ecalyculata & 0,21 & \\
\hline 12 & Cupania vernalis & 4,12 & \\
\hline 13 & Dalbergia frutescens & 0,41 & \\
\hline 14 & Erythrina falcata & 0,41 & \\
\hline 15 & Eugenia rostrifolia & 0,82 & \\
\hline 16 & Eugenia uniflora & 3,09 & \\
\hline 17 & Fagara rhoifolia & 0,21 & \\
\hline 18 & Helietta apiculata & 0,21 & \\
\hline 19 & Inga marginata & 6,60 & \\
\hline 20 & Luehea divaricata & 1,24 & \\
\hline 21 & Maba inconstans & 2,68 & \\
\hline 22 & Machaerium stipitatum & 1,65 & \\
\hline 23 & Manihot grahami & 0,41 & \\
\hline 24 & Matayba elaeagnoides & 2,27 & \\
\hline 25 & Maytenus ilicifolia & 0,21 & \\
\hline 26 & Myrocarpus frondosus & 3,30 & \\
\hline 27 & Nectandra megapotamica & 1,65 & \\
\hline 28 & ocotea puberula & 0,42 & \\
\hline 29 & Parapiptadenia rigida & 8,04 & \\
\hline 30 & Patagonula americana & 4,74 & \\
\hline 31 & Sebastiania brasiziensis & 0,62 & \\
\hline 32 & Sebastiania klotzchiana & 0,21 & \\
\hline 33 & Seguieria guaranitica & 1,03 & \\
\hline 34 & Solanum verbascifolium & 0,62 & \\
\hline 35 & Sorocea bomplandii & 1,44 & \\
\hline 36 & Strychnos brasiliensis & 0,21 & \\
\hline
\end{tabular}


TABELA III. Continuação.

\begin{tabular}{clc}
\hline \hline No & \multicolumn{1}{c}{ ESPECIES } & ABUNDANCIA RELATIVA \\
\hline 37 & Tecoma stans & 1,65 \\
38 & Trema micrantha & 0,41 \\
39 & Trichilia catigua & 8,04 \\
40 & Trichilia elegans & 1,86 \\
41 & Vizzaresia paniculata & 0,21 \\
42 & Não identificadas & 0,19 \\
\hline T 0 T A L & 100 \\
\hline
\end{tabular}

CONCLUSOES

A vegetação de Capoeira é altamente heterogēnea na região do curso médio do Rio Jacuí, RS, apresentando grandes diferenças quan to à estrutura, fisionomia e composição florística. Neste tipo flo restal são características as espécies pioneiras, tīpicas dos estā gios iniciais da sucessão vegetal. As espécies arbóreas constituin tes da mata original são encontradas com menor abundância e repre sentadas por individuos jovens.

\section{ZUSAMMENFASSUNG}

MARCHIORI,J.N.C.; LONGHI,S.J. und DURLO,M.A. Die Pioniervegetation (capoeira) in dem Gebiet der Mittelstrecke des Jacuitals, RS. Ciência e Natura (4):141-150.

Die vorliengende Arbeit bescreibt die floristihche Zusam mensetzung und die Struktur der Pioniervegetation (Capoeira) in dem Gebiet der Mittelstrecke des Jacuital.s in dem Bundesstaat Rio Grande do Sul. Diese Waldart weist viele Baum-und Sträucherarten auf. Es wurden 45 holzingen Species identifiziert, von denen die Mehrzahl als Pionierbaumarten einzuordnen sind. Die Bäume mit wirtschaftlicher Bedeutung kommten nicht häufig vor. Es wurden auch 96 nicht holzingen Species erkannt.

\section{REFERENCIAS BIBLIOGRAFICAS}

1. CAINE,S.A. \& CASTRO,G.M. de 0. Application of some phytosociological techniques to Brazilian Rain Forest. American Joumal of Botany, 43 (3):205-217, 1956.

2. FINOL,U.H. Nuevos parāmetros a considerar-se en el anālisis es tructural de las selvas virgenes tropicales. Rev. For.Venez., 14 (21):29-42, 1971.

3. FÖRSTER,M. Strukturanalyses ein es tropischen Regenwaldes in Kolumbien. Alzg. Forst. - u. J. - Ztg., 144(1):1-8, 1973. 
4. GOLDSTEIN,R.S. \& GP.INGAL,D.F. Definition of vegetation structure by canonical analysis. J. Ecol., 62 (2):227-84, 1972.

5. HUECK,K. As florestas da América do Sur. São Paulo, Polígono. 1972 $466 \mathrm{p}$.

6. KELLMAN,M.C. Plant geography. London, Methuen, 1975. 135 p.

7. LAMPRECHT, H. Ensayo sobre unos metodos para el Anālisis Estructu ral de los bosques tropicales. Acta Cientifica Venezolana, 13 (2): 57-65, 1962 .

8. MONTOYA MAQUIN, J.M. El acuerdo de Yangambi (1956) como base para una nomenclatura de tipos de vegetaciōn en el trōpico america no. Turrialba, 16 (2):169-180, 1966.

Recebido em agosto, 1982; aceito em outubro, 1982. 